\title{
Interpretation of the Protocol for Prevention and Control of COVID-19 in China (Edition 7)
}

\author{
Canjun Zheng'; Zhaorui Chang'; Fengfeng Liu'; Mengjie Geng'; Hui Chen²; Jingwei Jiang; \\ Sheng Zhou'; Lu Ran'; Zhongjie Li' ${ }^{1}$; Zijian Feng'; George F. Gao'; Liping Wang ${ }^{1, *}$
}

\section{INTRODUCTION}

After coronavirus disease 2019 (COVID-19), previously known as pneumonia of unknown etiology (PUE) and 2019 novel coronavirus (2019-nCoV), was first discovered in Wuhan City, Hubei Province, the Chinese government took measures to stop the spread of the epidemic nationwide and interrupted local transmission in China (1-2). Although there have been several localized outbreaks that were caused by overseas importation of the virus - including Heilongjiang, Jilin, Beijing, Liaoning, and other provincial-level administrative divisions (PLADs) - these outbreaks have been stopped within two to four weeks and were limited in scope to less than ten cases or a few hundred cases for each event. With the COVID-19 pandemic still uncontrolled globally, the threat of importing the virus continues unabated and still threatens China's population, virtually all of which is susceptible.

Non-pharmaceutical measures that effectively contained COVID-19 and stopped outbreaks due to importation were described in previous editions of the National Health Commission's (NHC) Protocol for Prevention and Control of COVID-19. The NHC and external experts cooperated to update this Protocol to a seventh edition to ensure effective responses to outbreaks and to a possible epidemic of COVID-19 this autumn and winter and to include experiences gained domestically, globally, and through research. This article described the primary content and key updates in the Protocol, including surveillance, multimethod monitoring, early warnings, and close contact tracing and management and discusses the scientific rationale for revisions of the protocol.

\section{EPIDEMIOLOGICAL CHARACTERISTICS}

Respiratory droplets and close contact are still considered the primary routes of COVID-19 transmission, but the new edition indicates that contact with virus-contaminated products can also cause infection (3). COVID-19 virus is sensitive to heat, but remains viable at low temperatures and can survive for an indeterminate period of time in low temperature environments, especially, as has been seen, in frozen foods and packaging. Investigations of the Beijing, Dalian, and Qingdao outbreaks showed that contaminated imported frozen foods were sources of the outbreak viruses. Edition 7 notifies localities of the need to monitor low-temperature environments for COVID-19, especially in frozen foods, to prevent similar epidemics.

\section{CASE SURVEILLANCE}

At present, COVID-19 transmission has essentially been disrupted in China (4), and strengthening case surveillance and early detection of COVID-19 is the top priority of the current control and prevention strategies. The revised protocol clearly requires strengthening of case surveillance in medical institutions, communities, schools, etc., to further increase the sensitivity of case detection.

In medical institutions, Edition 7 expands the nucleic acid testing requirements. Patients with respiratory symptoms like fever and dry cough who have an epidemiological history should undergo nucleic acid testing. However, patients without an epidemiological history who have respiratory symptoms like fever and dry cough, have engaged in cold chain food processing or sales, or have been at a farmers market, institutions for the elderly or disabled, a hospital, a detention center, a nursing home, or a school should undergo nucleic acid testing for the COVID-19. In addition to the relatively recentlydiscovered transmission potential from contaminated frozen foods, many COVID-19 outbreaks have occurred in crowded and enclosed places, such as welfare institutions, places of detention, nursing homes, and schools. Therefore, routine nucleic acid testing of patients from these locations is conducive to 
early detection of COVID-19 and outbreak prevention.

For communities, management staff must monitor the health of key populations, including individuals discharged from hospitals or quarantine, close contacts of quarantined individuals, and people entering China who completed quarantine. Experience from the initial containment effort in China showed that $10 \%$ to $12 \%$ of patients with COVID-19 tested positive after discharge. Although transmission from positive-testing, discharged patients has not been observed in China, some people who entered China tested positive after being released from isolation. To ensure discovery of sources of infection as much as possible, Edition 7 requires health monitoring of people returning to communities from high-risk settings such as quarantine or a COVID-19 hospital.

\section{CLUSTERS}

Presence of a cluster of cases is used as an indicator for categorizing geographic areas by risk - low, medium, and high. County and district sizes vary greatly in China, with populations ranging from tens of thousands to millions of people. For precise control and prevention measures that reasonably categorize medium- and high-risk areas, Edition 7 modifies the definition of a cluster to be " 5 or more cases found within a small area such as a school, residential community, factory, village, or medical institution within a 14-day period." This is a change from Edition 6 (5), which used a cutoff of two or more cases within 14 days. The new cluster definition increases the threshold for taking strict prevention and control measures. For example, if 2 cases were seen in a family in a county with millions of people, the Edition 6 definition would impact everyone in the county, whereas Edition 7 would not.

\section{MONITORING OF COLD-CHAIN FOOD PRODUCTS}

Outbreaks of COVID-19 have been documented among workers who process and handle frozen seafood domestically and overseas. China Customs has found multiple instances of COVID-19 nucleic acid positive samples from the outer packaging of frozen seafood and meat and from the outer packaging of frozen food sold in farmers markets. To address this importation risk, Edition 7 strengthens monitoring of cold-chain food products, farmers markets, and related business and their personnel. The purpose of this strengthening is to refine risk assessment of COVID-19 transmission, to improve environmental sanitation conditions for the production, processing, and trading of these products, and to improve everyday use of recommended protective measures.

\section{MULTI-METHOD MONITORING}

Edition 7 has a new section on multi-method monitoring and early warning. Relevant agencies are required to conduct fever monitoring in transportation and public service locations including airports, railway stations and wharfs, and shopping malls; kindergartens and schools must conduct absenteeism and symptom monitoring; pharmacies will monitor the designated drug sales; farmers markets and medical institutions will conduct environmental and personnel monitoring; and cold-chain food businesses will monitor their environment and personnel, as described above. Monitoring data will be collected in health department monitoring and early warning information platforms. Timely and comprehensive analyses of these data reflect risk of local epidemics and will help detect COVID-19 cases and asymptomatic infections, contaminated food, and coronavirus mutations as early as possible. Accurate assessment and the timely release of early warning information will better inform society and improve response times.

\section{CLOSE CONTACTS MANAGEMENT}

The seventh edition updates management of close contacts. Edition 6 did not require close contacts with no symptom to undergo nucleic acid testing during quarantine, but Edition 7 requires 3 nucleic acid tests during quarantine of close contacts. The first nucleic acid test is to be done within a day of starting centralized quarantine and medical observation, and the second test is done with a 1-day interval following the first. The third test is on the last day of the 14-day quarantine. The first two closely-spaced nucleic acid tests increase sensitivity for early detection of infected individuals to prevent transmission and provide timely treatment. Quarantine experience to date has found that many close contacts test positive or are ill at the beginning of quarantine and that some patients have longer incubation periods, sometimes more than 14 days. The timing of the third nucleic acid test is to 
reduce the likelihood of discharging an infected individual.

Edition 7 defines a new risk group - close contacts of close contacts (i.e., secondary contacts). Secondary contacts are to be identified through tracing and then managed for 14 days of centralized quarantine. This new requirement is based on several observations. Close contact tracing has been able to identify less than $40 \%$ of all cases, and over $60 \%$ of cases were not brought into quarantined management in time. During investigation of the Beijing and Jilin COVID19 outbreaks, we found that there were instances in which there were lengthy times between onset, detection, and isolated management - sometime more than 10 days. In such circumstances, close contacts may have been infected and been transmitting virus to their close contacts. The rationale for defining this new risk group is to find as many sources of infection as possible to stop the outbreak as rapidly as possible. Identification of secondary contacts, therefore, should identify more individuals at risk of infection.

\section{TRAVELERS INTO CHINA}

Edition 6, released in March 2020, did not specify management of travelers coming into China. At the time of Edition 6 being released, imported cases were not a significant proportion of cases. Starting in April, the NHC began to require people entering China from other countries to be quarantined for 14 days at the point of entry. Currently, the global pandemic is still severe but with the containment of COVID-19 in China, work and production have resumed and the number of people entering China has increased. Edition 7 adjusts management of travelers entering China to " $7+7$ " and " $2+1$ " strategies: " $7+7$ " strategy means that people entering China have a required centralized quarantine and medical observation at the point of entry for 7 days, and individuals with a negative nucleic acid test can continue quarantine at home for 7 more days; " $2+1$ " means that the entering traveler will undergo nucleic acid testing at the port of entry and again on the Day 7 of centralized quarantine. After two negative nucleic acid tests, the individual can quarantine at home and be tested again on Day 7-the last day of home quarantine. The use of the " $7+7$ " and " $2+1$ " methods for quarantine can accommodate more people and make quarantine more palatable, promoting the reopening of international flights and land borders. Thus far, there has been no documented transmission in areas adopting these two strategies.

\section{EPIDEMIC RESPONSE}

Edition 7 clarifies the scope and timing of prevention and control measures based on results of risk assessments. In medium-risk areas, the scope of prevention and control areas is delimited by school classes, building units, factory workshops, offices, and families as the smallest units. In high-risk areas, schools, buildings, factories, workplaces, and villages are the smallest units to delimit the scope of prevention and control areas. Edition 7 also clarifies specific measures to be taken in areas having different risk assessments. These adjustments are used to promote precise scientific prevention and control measures to minimize the impact of the measures on social and economic development and normal life for residents.

\section{MENTAL HEALTH SERVICES}

Mental health services are new inclusions in Edition 7. The COVID-19 pandemic is said to be a once-in-acentury public health emergency (6). The pandemic's severity and complexity have caused great social and psychological impacts on community residents, especially patients, quarantined people and their families, front-line disease prevention and control workers, and the elderly. Social and psychological stress leads to additional problems and adverse outcomes. Edition 7 requires relevant departments to jointly form psychological counseling and social work service teams that provide psychological counseling and assistance for key groups, including patients, people in quarantine, front-line workers, and the elderly. Services include psychological assistance hotlines, online psychological service platforms, and onsite counseling, all intended to promote physical and psychological recovery, a return to normal life and work, and social stability.

\section{DISCUSSION}

Edition 7 of the Protocol is based on the current domestic and international epidemic situations, new understandings of COVID-19, and the overall strategy of timely detection, rapid response, precise management, and interruption of transmission. It emphasizes strengthening case surveillance and multimethod monitoring, including sampling and testing of cold-chain food products and at-risk cold-chain 
workers, with the objective of detecting all possible sources of infection in time and increasing overall surveillance sensitivity. Edition 7 revises the definition of a cluster, streamlines delineation of control and prevention areas, and carefully relaxes management of persons entering China - changes made to reduce the impact on the normal social lives of residents and promote socioeconomic development while maintaining highly effective prevention and control of COVID-19.

As the science on COVID-19 advances at home and abroad, the epidemic situation in China evolves, and vaccines, diagnostics, and drugs are developed, the NHC's Protocol for Prevention and Control of COVID-19 will be updated to always provide the best possible technical support for achieving China's prevention and control targets.

Funding: This work was supported by China Ministry of Science and Technology (grant no. 2018ZX10713001), National Natural Science Foundation of China (No. 91846302).

doi: $10.46234 / \mathrm{ccdcw} 2020.245$

\# Corresponding author: Liping Wang, wanglp@chinacdc.cn.

${ }^{1}$ Division of Infectious Disease, Key Laboratory of Surveillance and Early Warning on Infectious Disease, China CDC, Beijing, China; ${ }^{2}$ National Center for Tuberculosis Control and Prevention, China
CDC, Beijing, China; ${ }^{3}$ National Institute for Communicable Disease Control and Prevention, China CDC, Beijing, China; ${ }^{4}$ Chinese Center for Disease Control and Prevention, Beijing, China.

Submitted: November 13, 2020; Accepted: November 18, 2020

\section{REFERENCES}

1. The 2019-nCoV Outbreak Joint Field Epidemiology Investigation Team, Li Q. Notes from the field: an outbreak of NCIP (2019-nCoV) infection in China-Wuhan, Hubei Province, 2019-2020. China CDC Wkly 2020;2(5):79 - 80. http://dx.doi.org/10.46234/ccdcw2020.022.

2. Li ZJ, Chen QL, Feng LZ, Rodewald L, Xia YY, Yu HL, et al. Active case finding with case management: the key to tackling the COVID-19 pandemic. Lancet 2020;396(10243):63 - 70. http://dx.doi.org/10.1016/ S0140-6736(20)31278-2.

3. Guo ZD, Wang ZY, Zhang SF, Li X, Li L, Li C, et al. Aerosol and surface distribution of severe acute respiratory syndrome coronavirus 2 in hospital wards, Wuhan, China, 2020. Emerg Infect Dis 2020;26(7): 1583 - 91. http://dx.doi.org/10.3201/eid2607.200885.

4. Zhou L, Wu ZY, Li ZJ, Zhang YP, McGoogan JM, Li Q, et al. One hundred days of coronavirus disease 2019 prevention and control in China. Clin Infect Dis 2020;ciaa725. http://dx.doi.org/10.1093/cid/ ciaa725.

5. National Health Commission of the People's Republic of China. Protocol for prevention and control of COVID-19 (Edition 6). China CDC Wkly 2020;2(19):321 - 6. http://dx.doi.org/10.46234/ccdcw2020. 082.

6. Wu ZY, McGoogan JM. Characteristics of and important lessons from the coronavirus disease 2019 (COVID-19) outbreak in China: summary of a report of 72,314 cases from the Chinese center for disease control and prevention. JAMA 2020;323(13):1239-42. http://dx.doi.org/ 10.1001/jama.2020.2648. 